\title{
Injecting Light of High-Power LEDs into Thin Light Guides
}

\author{
Hugo J. Cornelissen ${ }^{1}$, Chenhung $\mathrm{Ho}^{2}$, Haiyan $\mathrm{Ma}^{2}$, Marcel P.C.M. Krijn ${ }^{1}$, Hans A. van Sprang ${ }^{1}$ \\ ${ }^{1}$ Philips Research Europe, High Tech Campus 34, 5656AE Eindhoven, The Netherlands, \\ ${ }^{2}$ Optics Research Group, Delft University of Technology, the Netherlands
}

\begin{abstract}
A new method using a thin-film multilayer filter is described to couple light from high-power LEDs into a thin light guide such as an LCD backlight. Light emitted below the critical angle is reflected back to the LED and recycled. Largeangle emitted light passes the filter and is transported by total internal reflection in the light guide. The light guide can be as thin as $0.3 \mathrm{~mm}$ for an LED of $1 \times 1 \mathrm{~mm}^{2}$, and the best coupling efficiency is estimated to be around $80 \%$. With this approach, a backlight system can be greatly simplified but also compact collimators can be realized. In this paper the optical design and testing of the filter is described, and a $1 \mathrm{~mm}$ thick, $6.5 \mathrm{~mm}$ diameter collimator is presented that emits in a cone of $2 \times 13^{\circ}$. Measurements on prototypes show good agreement with the designed characteristics.
\end{abstract}

Keywords: LED, multilayer, interference filter, collimator, light guide

\section{INTRODUCTION}

The significant progress in the brightness and lumen efficiency of light-emitting diodes (LEDs) has made them become the most promising light source for liquid crystal display (LCD) backlights. Thin backlights for LCDs can be realized by coupling light at the edges of a flat or wedge-like light guide. By means of total internal reflection (TIR), the light from LEDs can be guided and transported in the light guides, and then coupled outwardly to the LC panel by various extraction structures on the light guides. For higher uniformity of the light output or for higher total light output, it is more attractive to distribute LEDs across the surface of the light guide. One of the conventional ways of doing this is by using side-emitting LEDs in recessions in the light guide [1]. However, there are several drawbacks to this technique, such as thick light guides $(3 \sim 6 \mathrm{~mm})$, the complicated structure and the precise alignment between LEDs and recessions, and the light shields placed above the LEDs to prevent light leakage.

The main objective of our new method is to overcome these drawbacks: the light can be coupled into a light guide without making any hole and recession in it. The new configuration is shown in Figure 1, where a Luxeon Rebel is the light source, a high-power blue-LED (peak wavelength: 460nm, FWHM: 24nm) manufactured by Philips Lumileds. This new-generation LED is equipped with a $1 \times 1 \mathrm{~mm}^{2}$ rough surface on the top of InGaN to increase the light extraction efficiency. The rough surface in conjunction with a mirror electrode behaves like a Lambertian reflector [2], which makes the scattered intensity distribution follow Lambert's cosine law. We remove the original overmolded silicone dome and bring the flat LED surface in optical contact with the light guide by means of a silicone adhesive.

The most important aspect of our method is the dielectric multilayer filter deposited on the bottom of the light guide [3]. The filter is optimized to only transmit light emitted from the LED at angles larger than the critical angle, $\theta_{\mathrm{c}}$ at the light guide-air interface. Most of the light emitted at smaller angles is reflected towards the rough InGaN surface and is recycled. The ideal filter performance is illustrated in the right part of Figure 1.

International Optical Design Conference 2010, edited by Julie Bentley, Anurag Gupta,

Richard N. Youngworth, Proc. of SPIE-OSA Vol. 7652, 765212 - @ 2010 SPIE

CCC code: $0277-786 \mathrm{X} / 10 / \$ 18 \cdot$ doi: $10.1117 / 12.870902$ 

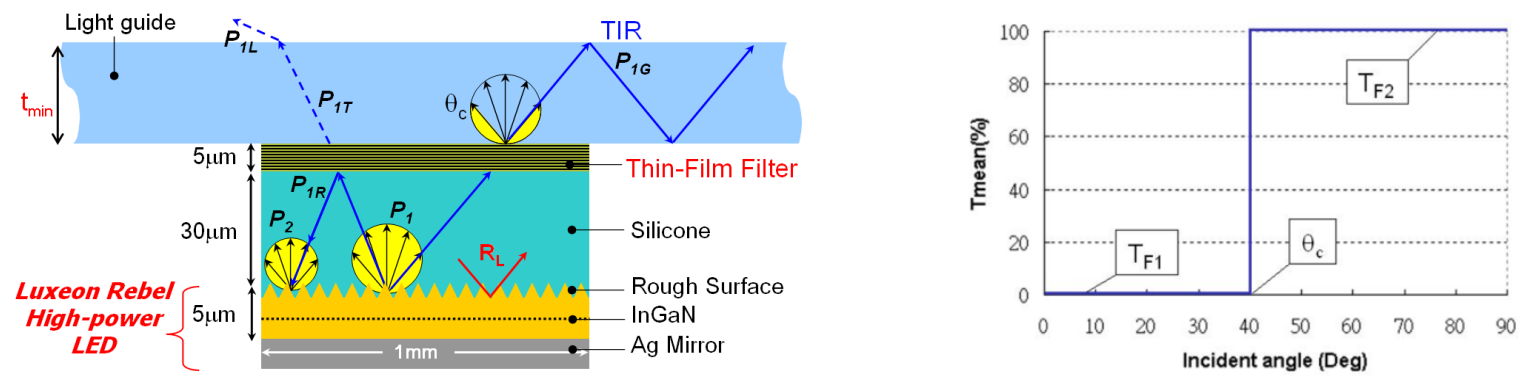

Figure 1: The new optical configuration for coupling LEDs to thin light guides (not drawn to scale). On the right the ideal performance is plotted of the filter applied to the whole spectrum of a certain LED.

\section{Thin film optics design of the multilayer angular filter}

In the design, the maximum number of layers was set at sixty layers and only two available materials were used, $\mathrm{Nb}_{2} \mathrm{O}_{5}$ and $\mathrm{SiO}_{2}$. Commercial software, Essential MacLeod [4], was used to optimize the performance of the filter. The ideal and targeted performance of the filter is $\left(T_{F 1}, T_{F 2}\right)=(0 \%, 100 \%)$ as shown in Figure 1 . With this filter, not only the coupling efficiency is maximized, but the leakage of light just above the LED is also minimized. In the thin-film optics design software, the average of s- and p-polarized transmission is optimized. Targets and weights were set for various parts of the LED spectrum and for various ranges of the incidence angle. In Figure 2 the angular dependent transmission of 460nm light is plotted of the final 54-layer design. Figure 3 zooms in on the angles of incidence smaller that the critical angles, and plots the transmission for various wavelengths. It is seen that in this angular range the transmission for this filter is well under $1 \%$.

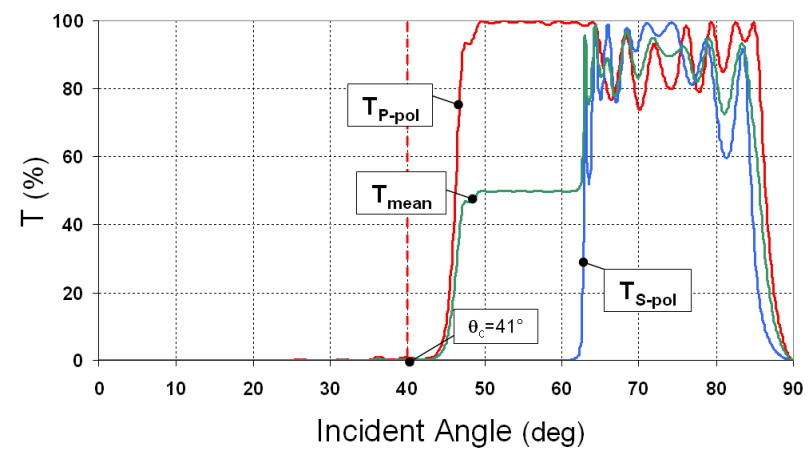

Figure 2 Calculated optimized transmittance at 460nm.Green curve: Mean Transmittance; Red curve: P-polarized

Transmittance; Blue curve: S-polarized Transmittance.

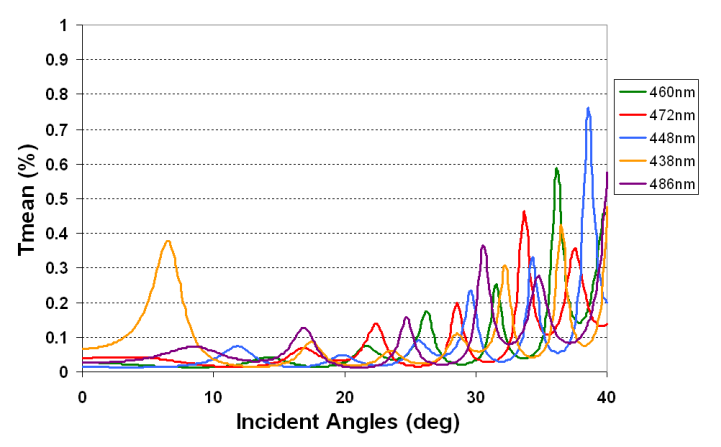

Figure 3 Calculated transmittance at five main wavelengths for small angle of incidence and with zoomed-in vertical scale.

\section{Optical characterization of the thin film stack}

The 54-layer filter was deposited by Philips Technology GmbH Global Technology Development (GTD Aachen). The spectral transmission of the filter was measured as a function of the incidence angle using a modified Perkin Elmer Spectrophotometer Lambda800 is shown in Figure 4. The filter is sandwiched between two cylindrical lenses with index matching oil and clipped on a rotational stage, which can be rotated manually to change the incident angle. The cylindrical lenses enable to measure at large incident angles, as the incident material and substrate are both glass in the filter design. The set-up was modeled in the ray tracing program ASAP to study the effect of the beam divergence and the radius of the cylindrical lens, see Figure 5. 


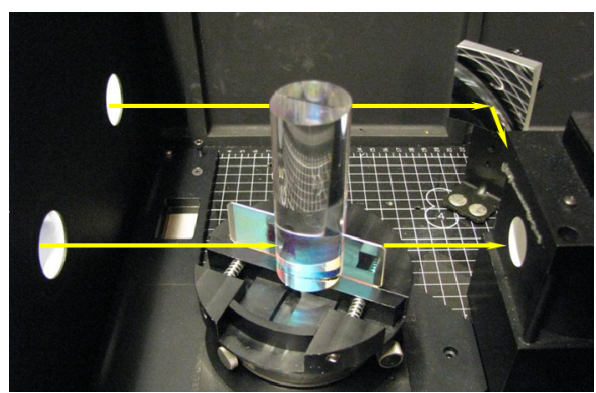

Figure 4 Modified Perkin Elmer Spectrophotometer Lambda800.

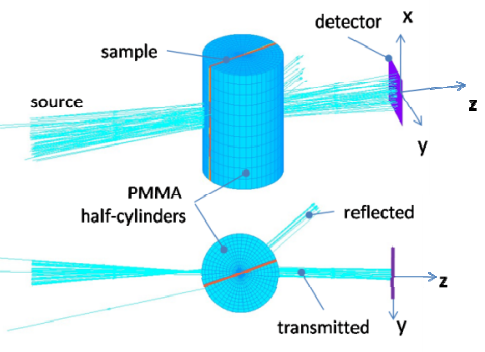

Figure $53 \mathrm{D}$ and projected view of the measurement setup simulated in ASAP to understand the influence of beam divergence and the cylindrical lens.

As shown in Figure 6, at incidence angles between $0^{\circ}$ and $40^{\circ}$, all LED light is reflected. At $50^{\circ}$ and $60^{\circ} 50 \%$ is transmitted (all p-polarized) and at $70^{\circ}$ around $80 \%$ transmission is measured. The calculated spectra show more peaks and structure that the measured ones. This can be explained by taking into account a beam divergence in the measurement set-up of several degrees in horizontal and vertical direction.
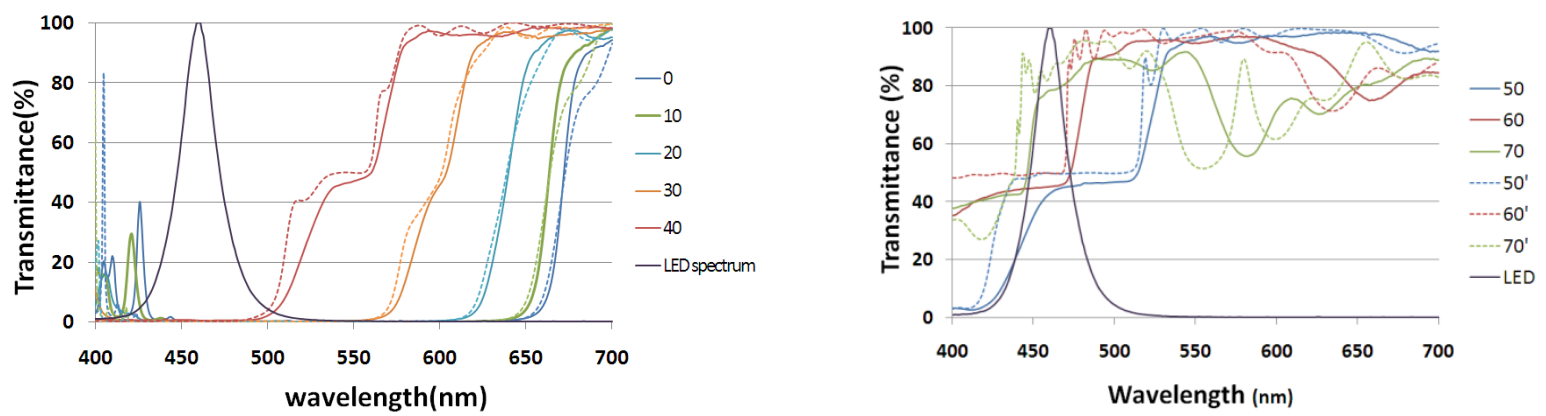

Figure 6 Measured and calculated mean transmittance with incident angles from $0^{\circ}$ to $40^{\circ}$ (left) and $50^{\circ}$ to $70^{\circ}$ (right). Dashed lines give calculated values, solid lines are the experiments. The Blue LED spectrum is shown to peak at $460 \mathrm{~nm}$.

\section{Test device}

To test the performance of the interference filter, interference filter stacks were deposited on glass sheets of $150 \mu \mathrm{m}$ thickness. Pieces of $1.8 \times 1.8 \mathrm{~mm}^{2}$ square were mounted on blue Luxeon Rebel LEDs. On top of the filter glass, $4 \mathrm{~mm}$ diameter glass domes were glued, as schematically drawn in Figure 7. In Figure 8 microscope pictures show that under ambient illumination the filter reflects all light and the LED appears like a mirror and hence dark under the microscope. When switched on, some blue emission is leaking through the filter. As a reference a standard Luxeon Rebel is pictured.

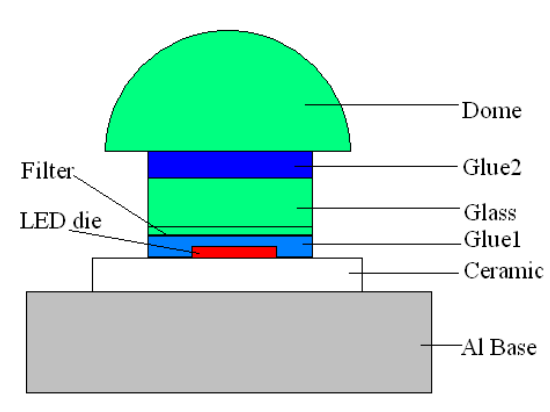

Figure 7 Schematic drawing of the test device.

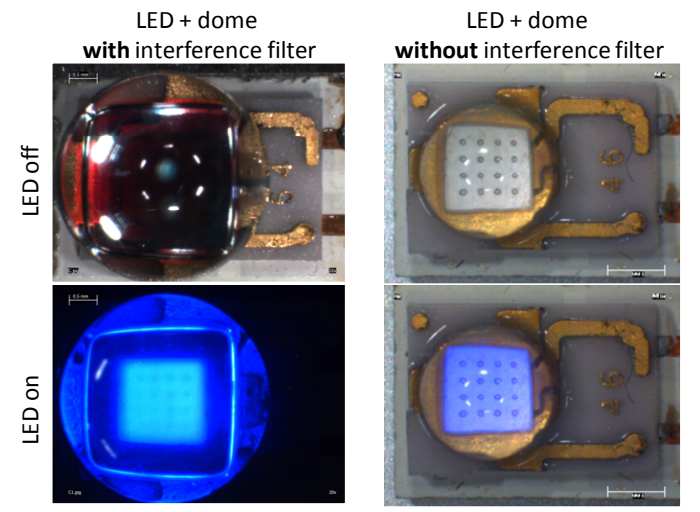

Figure 8 Microscope pictures of the test device (left) and of a standard blue Rebel (right) 
The luminous intensity of the prototype is measured with a Source Imaging Goniometer from Radiant Imaging and a comparison with the results from a LightTools model is shown in Figure 9 and Figure 10. As predicted the emission takes place in an angular ring at angles between approximately $45^{\circ}$ and $65^{\circ}$. At angles below $40^{\circ}$ a higher intensity is measured than predicted. This can be attributed to light leakage through the edges of the glass that contains the filter, as can be seen as bright lines in the lower left picture in Figure 8. When these edges are made absorbing, this low-angle light leakage is suppressed.
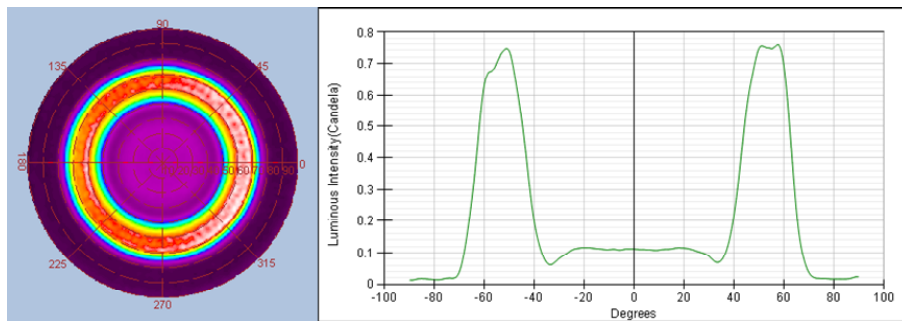

Figure 9 Measured luminous intensity distribution and profile.

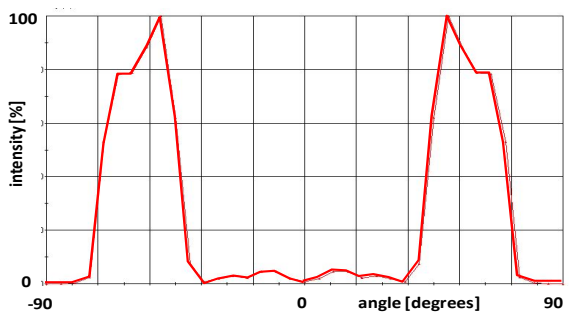

Figure 10 Calculated luminous intensity profile.

An important but unknown factor in the ray tracing modeling is the reflectivity of the LED for the recycled light. The light flux of the test devices as measured in an integrating sphere was observed to be approximately $50 \%$ compared to the reference LEDs. From fitting this measurement to the ray tracing modeling it is concluded that the reflectivity of the used Luxeon Rebel LEDs is approximately $65 \%$. The étendue of the reference LED, $G_{\text {ref }}$, is estimated as $G_{\text {ref }}=n^{2} \pi A=$ $7.1 \mathrm{~mm}^{2} \mathrm{sr}$, with $n=1.5$ the index of refraction and $A=1 \mathrm{~mm}^{2}$ the emitting surface area. For the LED with the interference filter the étendue estimate is, still inside the glass:

$$
G=n^{2} \pi A\left(\sin ^{2} 65^{\circ}-\sin ^{2} 45^{\circ}\right)=0.32 G_{\text {ref. }}
$$

So, the étendue is decreased but the flux in not fully maintained. Estimating the source luminance $L$ from the flux $\Phi$ and the étendue $G$ as $L=\Phi / G$, it can be expected that the luminance increases by a factor of $0.50 / 0.32=1.56$ due to the recycling. In a more conservative estimate, the upper angle of $65^{\circ}$ is replaced by $90^{\circ}$ and the étendue is reduced as $G=$ $0.50 G_{r e f}$. With a more ideal filter that transmits all angles from the critical angle upward to $90^{\circ}$ as shown in Figure 1, and a reflectivity of the LED surface of $65 \%$, the coupling efficiency can be calculated to be $79 \%$. If the LED reflectivity can be increased to $85 \%$, then the coupling efficiency approaches $90 \%$. From ray tracing it shows that the minimum thickness of the light guide, $t_{\min }$ in Figure 1, can be reduced to $300 \mu \mathrm{m}$ without a penalty in flux.

\section{Design of a compact collimator}

A very compact collimator can be designed based on the multilayer filter approach. A filter as deposited on a $1 \times 1 \mathrm{~mm}^{2}$ $150 \mu \mathrm{m}$ thick glass substrate is mounted on an LED with silicone adhesive. A circular disk light guide made from PMMA is cone shaped with a facet at $26^{\circ}$ angle, as illustrated in Figure 11, and mounted onto the filter glass. The PMMA piece is $1 \mathrm{~mm}$ thick and tapers from $2.5 \mathrm{~mm}$ to $6.5 \mathrm{~mm}$ diameter. The optical performance of the prototype is measured with a Source Imaging Goniometer from Radiant Imaging and favourably compares with the results from the LightTools model as shown in Figure 12.

Our approach provides a compact alternative to the method presented in [5], where light is recycled through the LED source using reflective micro-facets. In the present case thin film optics is applied, exploiting the relatively narrow spectral bandwidth of the LED emission.
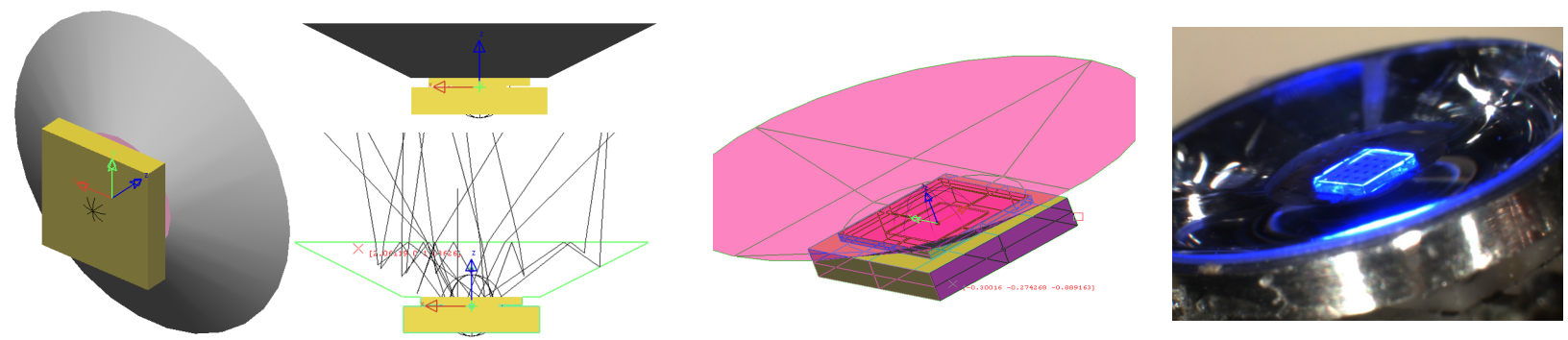

Figure 11 LightTools model of a compact collimator and a microscope picture of a prototype. 

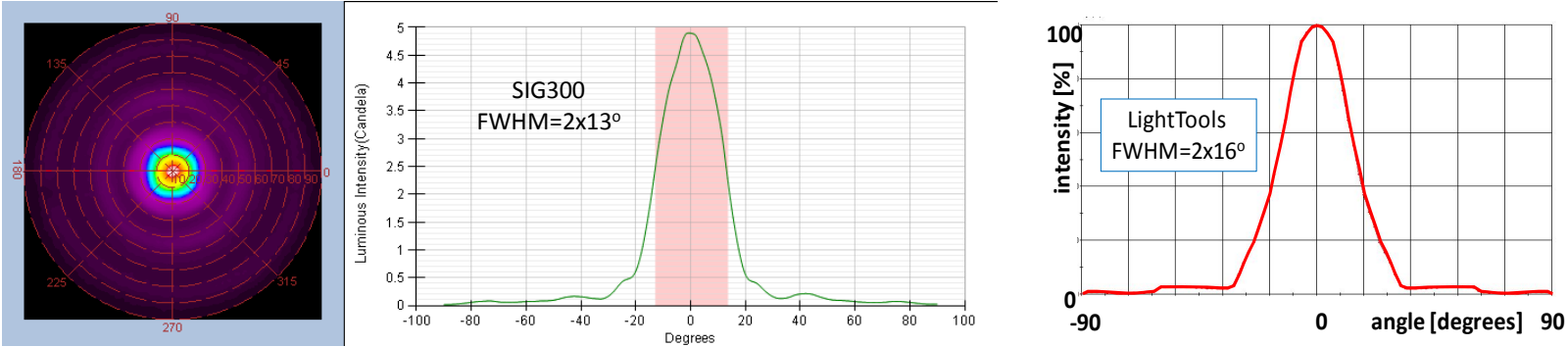

Figure 12 Optical characterization of the collimator prototype. The measured luminous intensity distribution is compared with the results from the LightTools model.

Looking into the source the spatial luminance distribution at perpendicular observation is shown in Figure 13 and a picture is given in Figure 14. The luminance distribution is calculated using backward ray tracing and shows good agreement with the experiment. The light is seen to emerge from a ring shaped area, not spanning the full exit area. If the full $6.5 \mathrm{~mm}$ diameter exit area were used, the étendue-limited Full Width at Half Maximum (FWHM) would be estimated as $2 \times 8.5^{\circ}$. Instead, as shown in Figure 12, the measured FWHM is $2 \times 13^{\circ}$, which suggests that the optical design of the collimator can be improved. This has been verified in ray tracing modeling, where the relatively simple single facet cone has been replaced by a more intricate curved shape. Furthermore it is expected that the reflectivity of the LED surface will be improved in the future, increasing the recycling efficiency.

The use of interference filters as angular recycling filters offers many interesting possibilities. For instance, restricting the transmission angular range of the filter will result in an even narrower collimation. The filter transmission characteristic might be improved by using more than just 2 materials in the interference stack. The concept is not limited to blue LEDs. It has already been proven that interference angular filters for red and green LEDs can be designed.
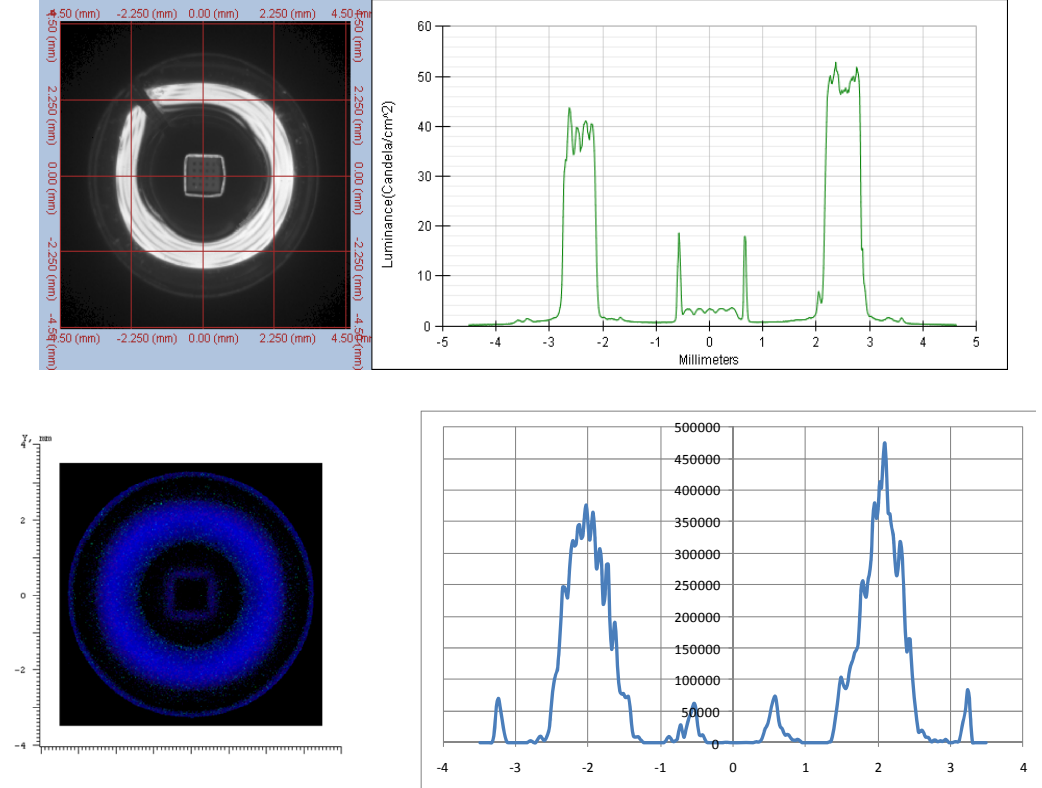

Figure 13 Spatial luminance distribution as observed along the normal, measured and calculated with backward ray tracing.

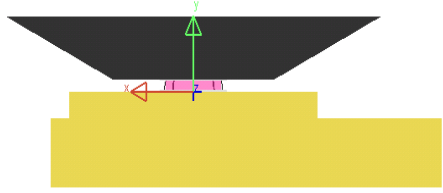

side view, schematic

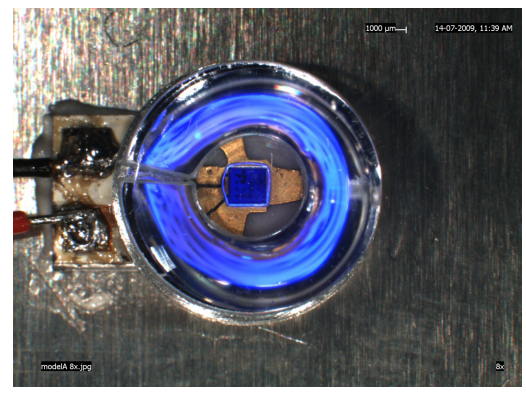

Figure 14 Picture of the collimator as observed along the normal, at low current for illustration purposes. 


\section{Acknowledgments}

We acknowledge the help of Kai Yuan Chi and Meijie Li in optical simulations. Serge Bierhuizen, Eugen Onac, Henri Jagt, Cor de Haas and Thomas Diederich are acknowledged for realizing the prototypes. We thank Florian Bociort and Paul Urbach for helpful discussions.

\section{References}

[1] R. S. West, H. Konijn, W. Sillevis-Smitt, S. Kuppens, N. Pfeffer, Y. Martynov, Y. Takaaki, S. Eberle, G. Harbers, "High brightness direct LED backlight for LCD-TV", SID 03 Digest, p. 1262.

[2] E. Fred Schubert, "Light Emitting Diode", 2nd ed., Cambridge Univ. Press (2006).

[3] C.H. Ho, H.J. Cornelissen, M.P.C.M. Krijn, and H.A. van Sprang, "Multilayer-Incoupling-Structure Design for Ultra-Thin LED-Backlights”, International Display Workshop 2008 IDW'08, 757-760 (2008).

[4] H.A. Macleod, “Thin-Film Optical Filters”, 3rd ed., Inst. of Phys., London, 2001.

[5] Julio C. Chaves, Waqidi Falicoff, Bill Parkyn, Pablo Benítez, Juan C. Miñano, "Increased brightness by light recirculation through an LED source", Nonimaging Optics and Efficient Illumination Systems V, Proc. of SPIE Vol. 7059 705902-1(2008) 